\title{
An Investigation on The Effect of Machine Gauge on the Properties of Weft Knitted Fabric
}

\author{
Mohammad Hosain Reza \\ Assistant Professor, Department of Textile Engineering, \\ Daffodil International University, Dhaka, Bangladesh \\ Md. Tanveer Ahmed \\ Director (Marketing), Southern Clothing Ltd., Zirabo, Savar, Dhaka, \\ Bangladesh
}

Doi: 10.19044/esj.2017.v13n33p354 URL:http://dx.doi.org/10.19044/esj.2017.v13n33p354

\begin{abstract}
To investigate the effect of machine gauge on properties of weft knitted fabric, three $\mathrm{V}$ - bed knitting machines of different gauges were selected for fabric sample production. They were of gauge 7, 12, and 14 . With these three machines, three $1 \times 1$ rib fabric samples were produced by using $20 / 2(\mathrm{Ne})$ yarn. Keeping the same machine settings, $1 \times 1$ rib fabric samples were produced by using $32 / 2(\mathrm{Ne})$ and $40 / 2(\mathrm{Ne})$ yarns. The dimensional properties like course / $\mathrm{cm}$, wales / $\mathrm{cm}$, stitch density per square $\mathrm{cm}$, and G.S.M. were measured from the samples. They were tabulated and presented by graphs to observe the effects. To analyze the effects, the stitch length of each fabric's samples was measured. It was found that the stitch length increases as the machine gauge decreases and vice-versa. As a result, all other properties like; course / cm, wales / cm, stitch density and G.S.M. also changes. It was concluded that the machine gauge affects the knit fabric properties and it has a specific trend.
\end{abstract}

Keywords: Knitted fabric, machine gauge, course / cm, wales / cm, stitch density, G.S.M. Dimensional properties

\section{Introduction}

The number of elements present in a unit length is called machine gauge. Generally, gauge means needles per inch. However, all primary knitting elements (like, needles) directly involved in the knitting action of a knitting machine is generally set to the same gauge. The pitch or distance between one needle to adjacent needles is inversely proportional to the needle gauge. The pitch (which is determined by the gauge) determines the yarn count or yarn thickness that can be knitted by the machine. Knitting 
machines with finer gauge tend to knit a wider fabric because more wales are produced in a unit length of fabric than a coarser gauge machine can produce. As the distance between the needles varies, the fabric that constitutes the stitch length also varies. As a result, there is also a variation in its properties.

\section{Experimental Details}

To find out the effect of machine gauge on weft knitted fabric properties, three different $\mathrm{V}$ - bed knitting machines of gauge 7,12 , and 14 were used to produce the fabric samples. Again to see whether the effects are applicable in case of varying yarn count, three different yarns (20 / 2 Ne, 32 / $2 \mathrm{Ne}$ and $40 / 2 \mathrm{Ne}$ ) were used to produce the samples in each machine. Finally, 9 samples were obtained (i.e. Each machine produced fabric samples from yarns of $20 / 2,32 / 2$ and $40 / 2$ counts.) For the experiment, 100\% cotton combed and plied yarns were used. These yarns are generally used to produce sweaters. It is mentionable that all produced fabric samples were $1 \times$ 1 rib structure. When knitting samples, the machine settings like yarn tension and fabric take down tension were kept same.

After knitting, the produced samples were left in relaxed position for 24 hours. After then, the properties course $/ \mathrm{cm}$, wales / cm, stitch density, and G.S.M. were tested according to the following procedure mentioned in sections 2.1, 2.2, 2.3, and 2.4. Again, the stitch lengths of the fabric samples were measured according to the section mentioned in 2.5.

The averages of tested data were then represented in graphs. From the graphs, the effects were noted.

\section{Course per $\mathrm{cm}$}

A course is a predominantly horizontal row of loops produced by the adjacent needles during the same knitting cycle. In brief, courses are rows of loops across the length of the fabric. It is measured along the wales direction by needle and counting glass. 10 readings were taken for course per $\mathrm{cm}$ from each sample. They were then averaged to get the final data.

\section{Wales per cm}

A Wale is a vertical column of intermeshed needle loops produced by the same needles knitting at successive knitting cycle. In brief, wales are columns of loops across the width of the fabric. It is measured along the course direction by needle and counting glass. 10 readings were taken for wales per $\mathrm{cm}$ from each sample. They were then averaged to get the final data. 


\section{Stitch Density}

Stitch density means the total number of loops in a measured area of fabric (per square inch or per square centimeter). It can be also obtained by counting number of courses per $\mathrm{cm}$ and number of wales per $\mathrm{cm}$, then multiplying them. Through the help of a counting glass, 10 readings were taken for stitch density from each sample. They were then averaged to get the final data.

\section{G.S.M.}

The property is self-explanatory which indicates the weight in grams, of one square meter fabric. To determine the G.S.M. of the fabric samples, the following procedure was followed:

a) A rectangular piece of fabric sample was cut from the main sample.

b) Its length and width were measured and recorded in nearest $\mathrm{mm}$.

c) The sample was then weighted by the help of a digital balance to the nearest gm.

d) Finally, the G.S.M. was calculated with the help of following formula:

G.S.M. $=\frac{\text { Sample weight in } \mathrm{gm}}{\text { Length } \times \text { width } \text { in } \mathrm{mm}} \times 1000 \times 1000$

\section{Stitch Length}

Stitch length is the length of yarn knitted into a complete stitch. A stitch is comprised of a needle loop and half of two sinker loops. To determine the stitch length, the following procedure was followed:

a) 20 loops were counted at the edge of the fabric, across the course direction.

b) Start and the end point of the 20 loops were marked on yarn which is bent into loops.

c) The loops were then unraveled and straitened to yarn.

d) The distance between the start and end marks on the yarn were measured in mm.

e) Finally, the lengths measured in mm were divided by 20 (number of loops) to obtain the stitch length. Through this way, 10 readings were taken for stitch length from each sample. They were then averaged to get the final data. 


\section{Result and Discussion}

Table 1. Values of fabric properties knitted with $20 / 2(\mathrm{Ne})$ yarn in different gauges

\begin{tabular}{|c|c|c|c|}
\hline Fabric property & \multicolumn{3}{|c|}{ Machine Gauge } \\
\hline & 7 & 12 & 14 \\
\hline Course / cm & 4.83 & 8.66 & 10.17 \\
\hline Wales / cm & 6.86 & 12 & 13 \\
\hline Stitch density & 33.13 & 103.92 & 131.8 \\
\hline G.S.M & 171.8 & 325 & 378 \\
\hline
\end{tabular}

Table 2. Values of fabric properties knitted with 32 / 2 (Ne) yarn in different gauges

\begin{tabular}{|c|c|c|c|}
\hline Fabric property & \multicolumn{3}{|c|}{ Machine Gauge } \\
\hline & 7 & 12 & 14 \\
\hline Course / cm & 4.74 & 8.01 & 9.68 \\
\hline Wales / cm & 6.22 & 13 & 13.56 \\
\hline Stitch density & 29.48 & 103.8 & 131.26 .8 \\
\hline G.S.M & 187.4 & 227.8 & 258 \\
\hline
\end{tabular}

Table 3. Values of fabric properties knitted with $40 / 2(\mathrm{Ne})$ yarn in different gauges

\begin{tabular}{|c|c|c|c|}
\hline & \multicolumn{3}{|c|}{ Machine Gauge } \\
\hline Fabric property & 7 & 12 & 14 \\
\hline Course / cm & 4.48 & 7.55 & 10.5 \\
\hline Wales / cm & 5.56 & 12.32 & 13.79 \\
\hline Stitch density & 24.9 & 93.01 & 144.795 \\
\hline G.S.M & 75 & 154.5 & 188 \\
\hline
\end{tabular}

Table 4. Stitch length ( $\mathrm{mm}$ ) of fabric knitted with different count in different gauges

\begin{tabular}{|c|c|c|c|}
\hline \multirow{2}{*}{ Yarn Count } & \multicolumn{3}{|c|}{ Machine Gauge } \\
\cline { 2 - 4 } & 7 & 12 & 14 \\
\cline { 2 - 4 } & \multicolumn{3}{|c|}{ Stitch length (mm) } \\
\hline $20 / 2$ & 9.47 & 5.24 & 4.81 \\
\hline $32 / 2$ & 9.353 & 5.11 & 5.04 \\
\hline $40 / 2$ & 9.12 & 5.3 & 5.09 \\
\hline
\end{tabular}




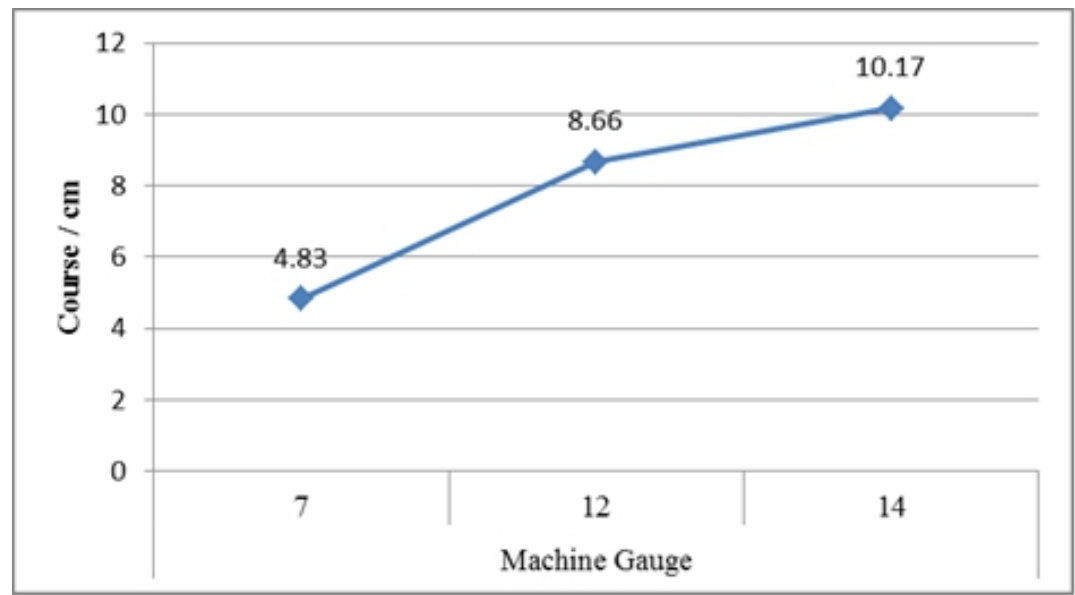

Figure 1. Course / cm of fabric knitted with $20 / 2$ (Ne) yarn in different gauges

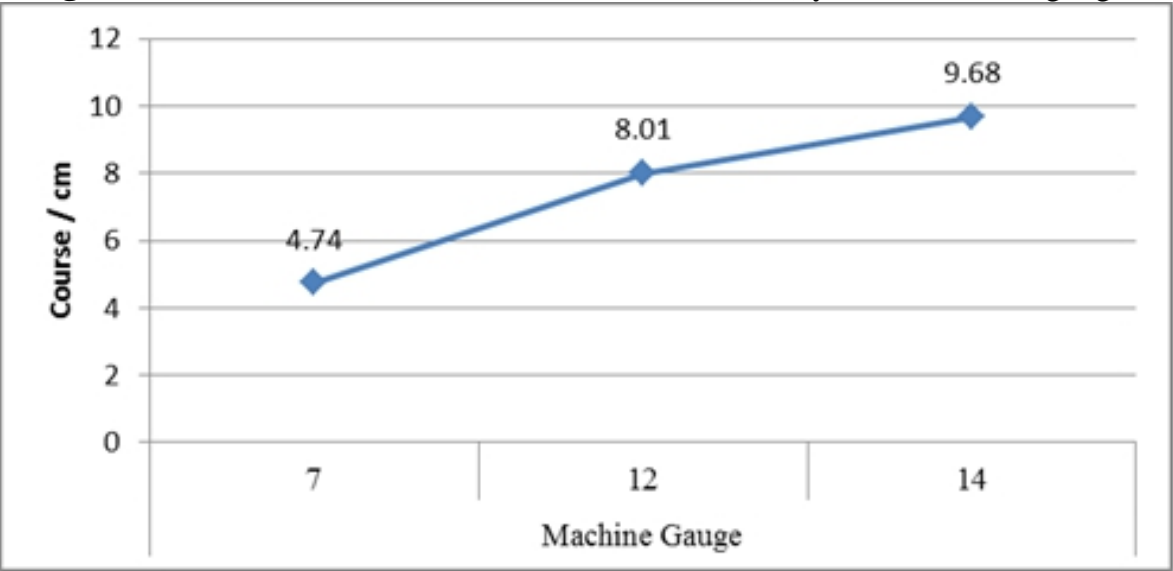

Figure 2. Course / cm of fabric knitted with $32 / 2$ (Ne) yarn in different gauges

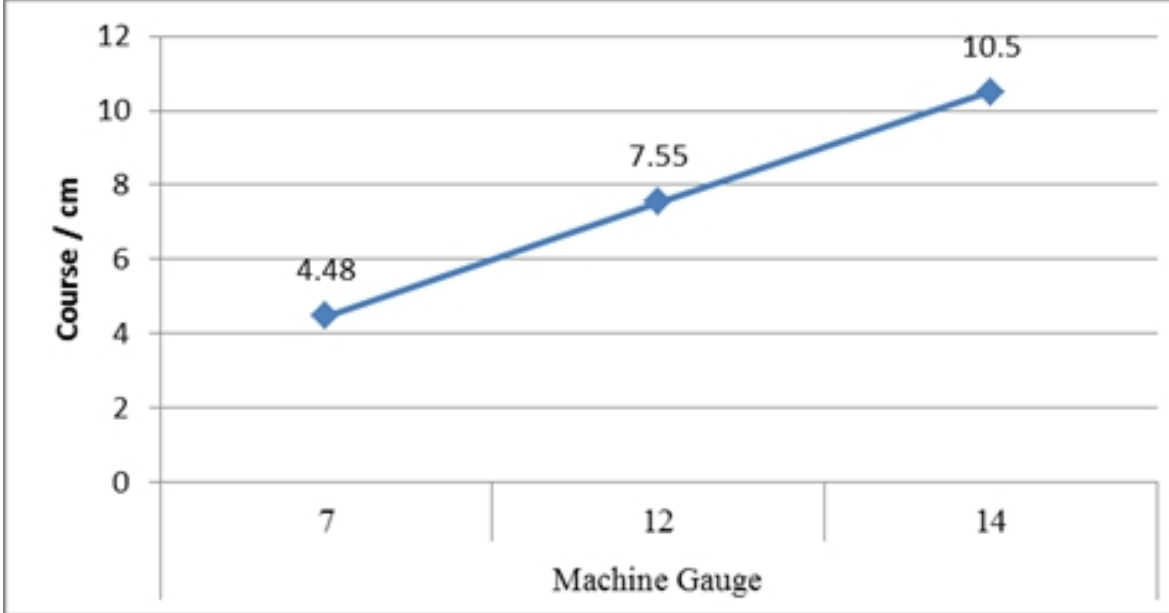

Figure 3. Course / cm of fabric knitted with 40 / 2 (Ne) yarn in different gauges 


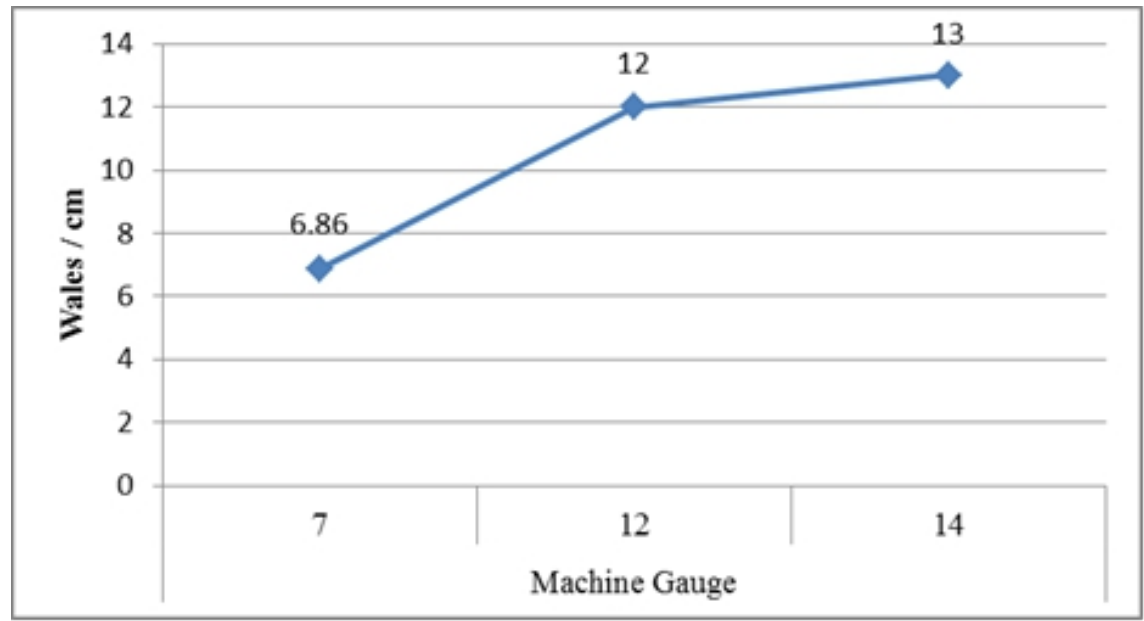

Figure 4. Wales / cm of fabric knitted with $20 / 2$ (Ne) yarn in different gauges.

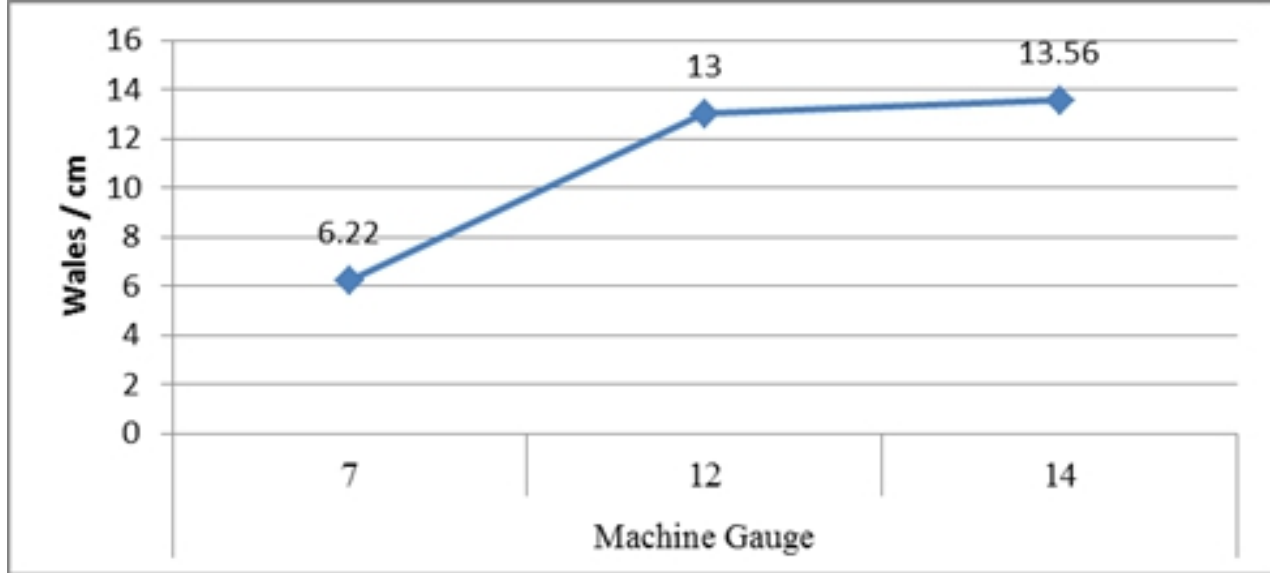

Figure 5. Wales / cm of fabric knitted with 32 / 2 (Ne) yarn in different gauges

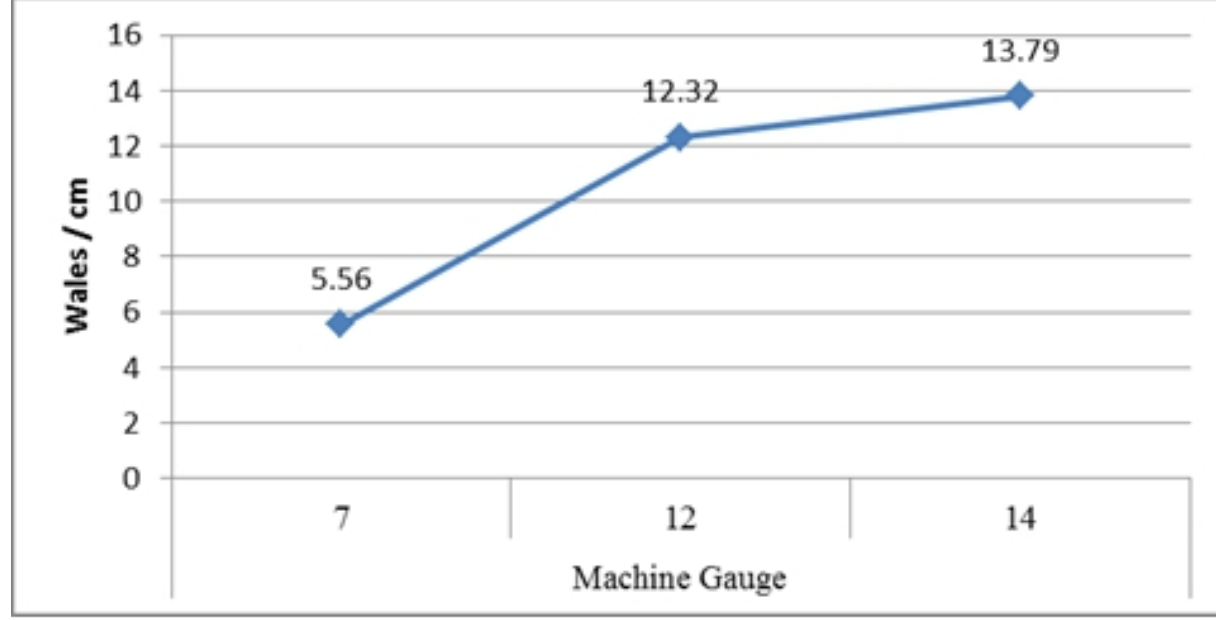

Figure 6. Wales / cm of fabric knitted with 40 / 2 (Ne) yarn in different gauges 


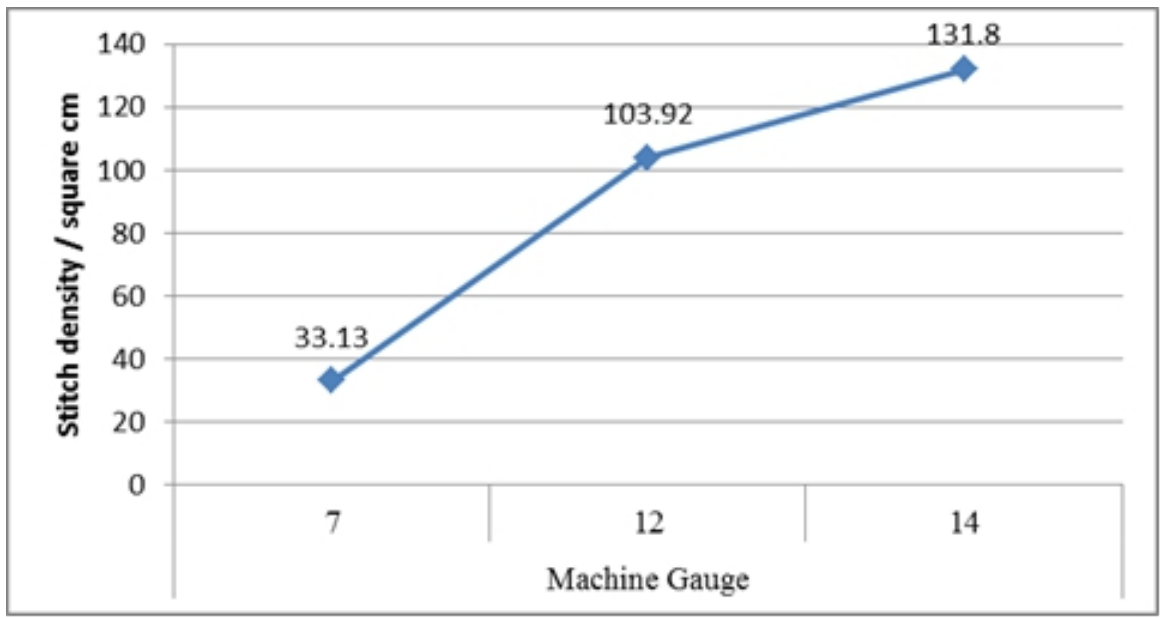

Figure 7. Stitch density / square cm of fabric knitted with 20 / 2 (Ne) yarn in different gauges

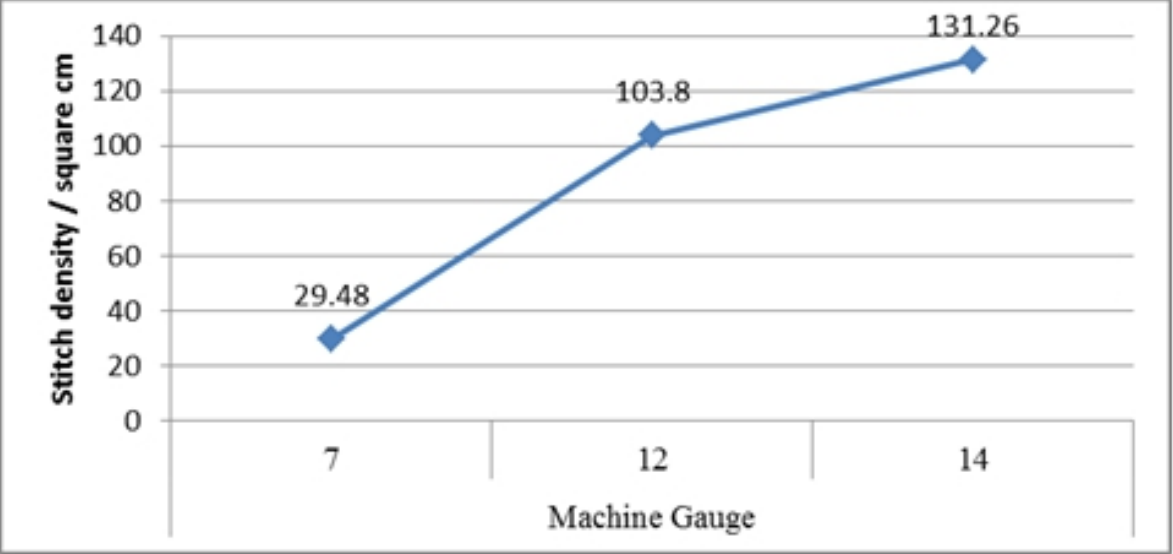

Figure 8. Stitch density / square cm of fabric knitted with 32 / $2(\mathrm{Ne})$ yarn in different gauges.

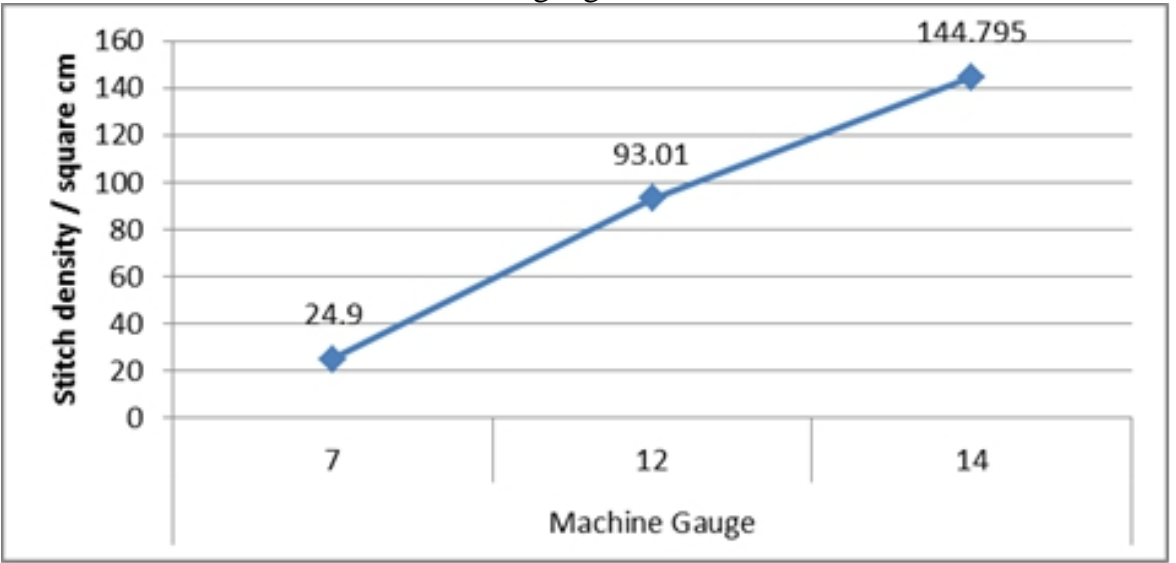

Figure 9. Stitch density / square cm of fabric knitted with 40 / 2 (Ne) yarn in different gauges 


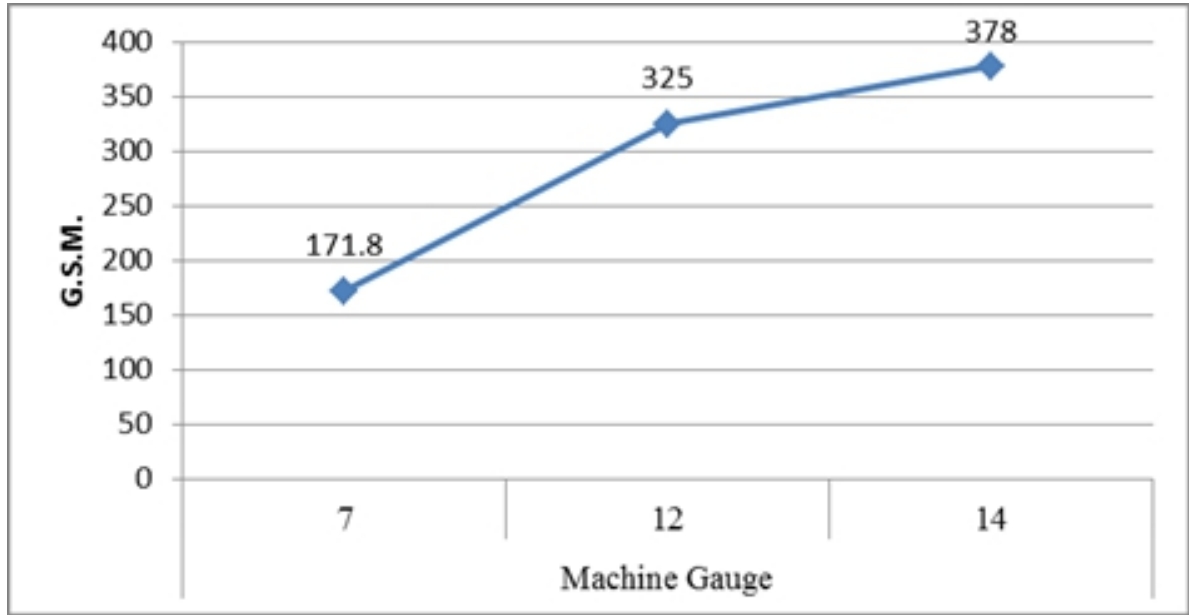

Figure 10. G.S.M. of fabric knitted with $20 / 2$ (Ne) yarn in different gauges

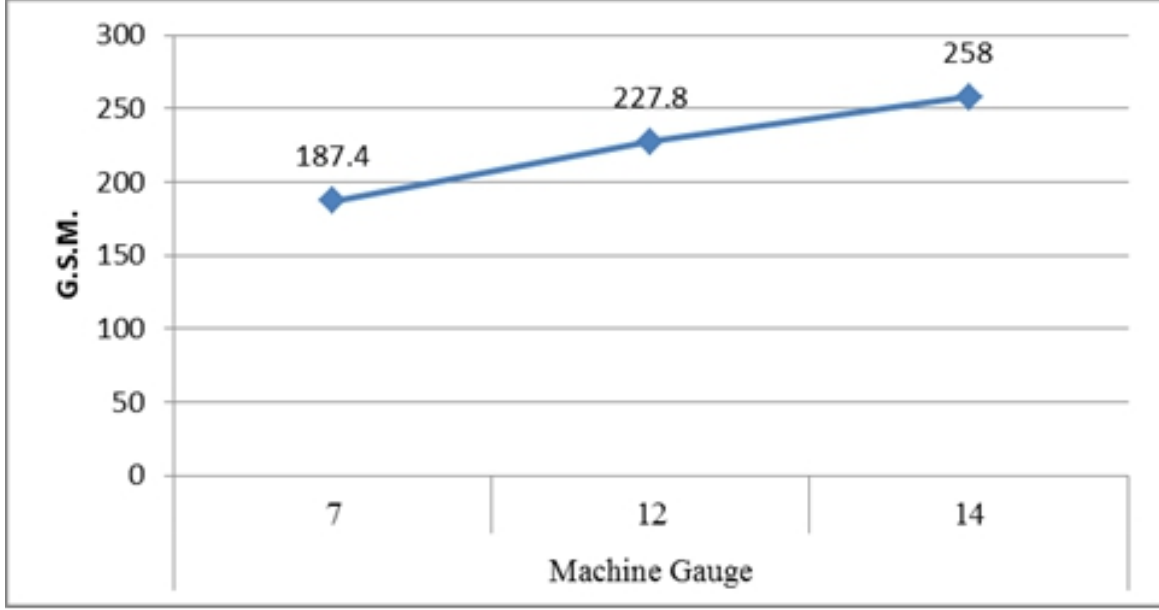

Figure 11. G.S.M. of fabric knitted with $32 / 2(\mathrm{Ne})$ yarn in different gauges

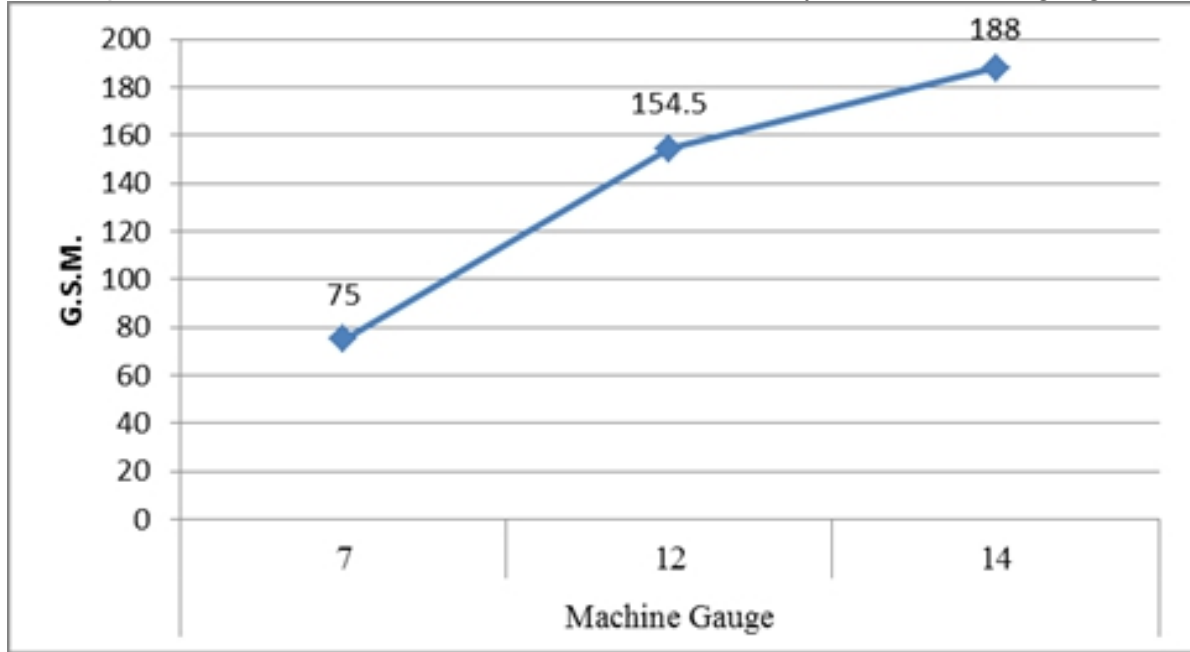

Figure 12. G.S.M. of fabric knitted with $40 / 2(\mathrm{Ne})$ yarn in different gauges 


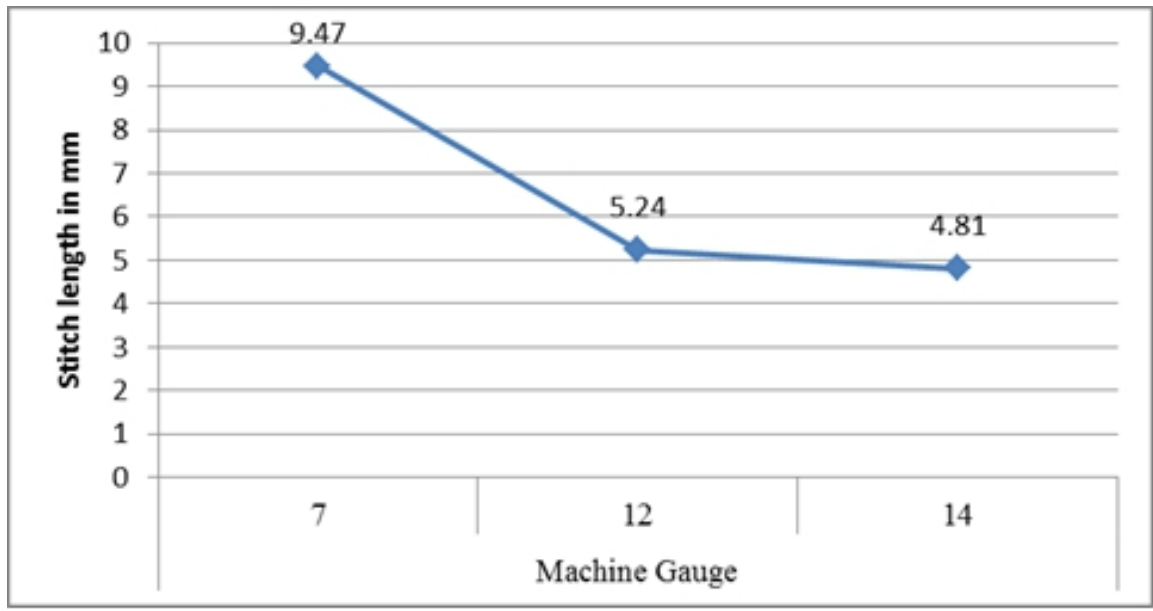

Figure 13. Stitch length $(\mathrm{mm})$ of fabric knitted with 20 / 2 (Ne) yarn in different gauges

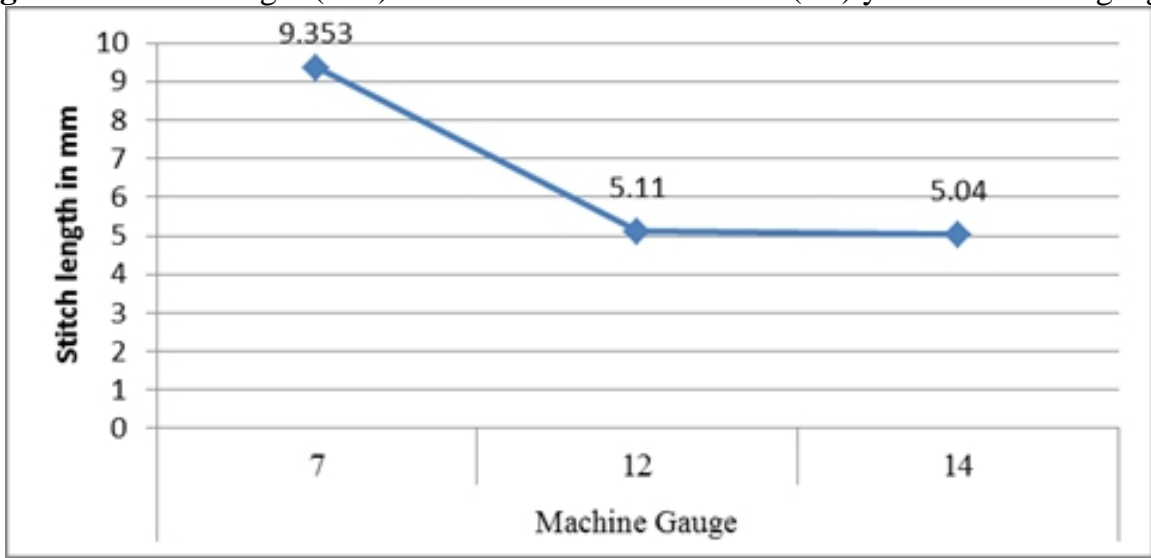

Figure 14. Stitch length $(\mathrm{mm})$ of fabric knitted with $32 / 2(\mathrm{Ne})$ yarn in different gauges

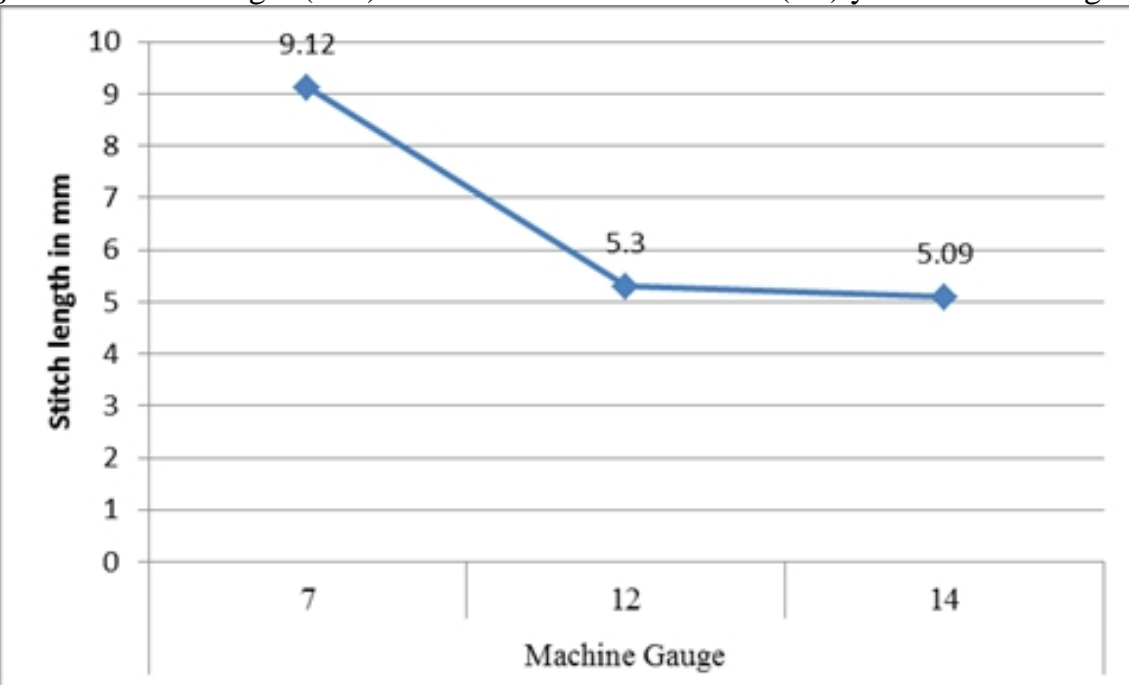

Figure 15. Stitch length (mm) of fabric knitted with 40 / 2 (Ne) yarn in different gauges 
From the figures $1-6$, it is clearly seen that with the increase of machine gauge, the course $/ \mathrm{cm}$ and wales $/ \mathrm{cm}$ have increased in every knit fabric samples. As a result, the stitches / square $\mathrm{cm}$ have also increased. This can be seen in figure $7-9$. Again in figure $10-12$, we can see that with the increase of machine gauge, the G.S.M. has increased in every fabric samples knitted from different yarn count.

From figures 13 - 15, it is found that the stitch lengths have decreased with the increase of machine gauge. Shorter stitch means shorter loops or stitches. So, more number of loops can accommodate per $\mathrm{cm}$ of fabric. As a result of decreased stitch length, the course/ $\mathrm{cm}$ and wales / $\mathrm{cm}$ have increased. It is obvious that the multiplication result of course / $\mathrm{cm}$ and wales / cm; i.e., stitch density per square $\mathrm{cm}$ will increase. Again, graphs can be interpreted in the opposite way i.e., vice-versa. This can be seen in Figure 7, 8 , and 9.

Finally, as the G.S.M. is inversely proportional to stitch length, it has increased with the decrease of stitch length (Figure 10 - 12).

\section{Conclusion}

In conclusion, a complete stitch is composed of a head and two side limbs or legs. The yarn passes from the foot of one loop into the foot and leg of the next loop formed by it. With the decrease of machine gauge, the pitch or distance between the adjacent needle increases. As a result, the distance between the adjacent loops also increases. This leads to lengthen the side limbs of the stitch. The final result is increased stitch length in coarser gauge machines; i.e., the stitch length increases when the machine gauge decreases. Here, it was found that the stitch length inversely increases or decreases with the machine gauge. As the properties like, course $/ \mathrm{cm}$, wales $/ \mathrm{cm}$, stitch density and G.S.M. is dependent on stitch length of the fabric, they also increase or decrease following their relationship with the stitch length.

\section{References:}

1. AmbumanI, N. (2007). "Knitting Fundamentals, Machines, Structures and Developments", First Edition, New Age International Publishers, New Delhi, India.

2. Booth, J.E. "Principles of Textile Testing", Third Edition, Newnes_Butterworths, London.

3. Iyer Mammel \& Schach (2004). "Circular Knitting”, Second Edition, Meisenbach GmbH, Bamberg, Germany.

4. Spencer David J. (2001). "Knitting Technology", Third Edition, Woodhead Publishing Limited, Cambridge, England. 